ISSN 2075-4701

www.mdpi.com/journal/metals/

Article

\title{
Phase Transitions in Mechanically Milled Mn-Al-C Permanent Magnets
}

\author{
Michael J. Lucis, Timothy E. Prost, Xiujuan Jiang, Meiyu Wang and Jeffrey E. Shield * \\ Department of Mechanical and Materials Engineering and Nebraska Center for Materials and \\ Nanoscience, University of Nebraska, Lincoln, NE 68588, USA; \\ E-Mails: mlucis@huskers.unl.edu (M.J.L.); tmthprost2@gmail.com (T.E.P.); \\ xiujuan.jiang@huskers.unl.edu (X.J.); meiyv.wang@gmail.com (M.W.) \\ * Author to whom correspondence should be addressed; E-Mail: jshield2@unl.edu; \\ Tel.: +1-402-472-2378; Fax: +1-402-472-1465.
}

Received: 30 January 2014; in revised form: 3 April 2014 / Accepted: 4 April 2014 / Published: 17 April 2014

\begin{abstract}
Mn}$-Al powders were prepared by rapid solidification followed by high-energy mechanical milling. The rapid solidification resulted in single-phase $\varepsilon$. The milling was performed in both the $\varepsilon$ phase and the $\tau$ phase, with the $\tau$-phase formation accomplished through a heat treatment at $500{ }^{\circ} \mathrm{C}$ for $10 \mathrm{~min}$. For the $\varepsilon$-milled samples, the conversion of the $\varepsilon$ to the $\tau$ phase was accomplished after milling via the same heat treatment. Mechanical milling induced a significant increase in coercivity in both cases, reaching $4.5 \mathrm{kOe}$ and $4.1 \mathrm{kOe}$, respectively, followed by a decrease upon further milling. The increase in coercivity was the result of grain refinement induced by the high-energy mechanical milling. Additionally, in both cases a loss in magnetization was observed. Milling in the $\varepsilon$ phase showed a smaller decrease in the magnetization due to a higher content of the $\tau$ phase. The loss in magnetization was attributed to a stress-induced transition to the equilibrium phases, as no site disorder or oxidation was observed. Surfactant-assisted milling in oleic acid also improved coercivity, but in this case values reached $>4 \mathrm{kOe}$ and remained stable at least through $32 \mathrm{~h}$ of milling.
\end{abstract}

Keywords: permanent magnets; mechanical milling 


\section{Introduction}

Mn-Al-based permanent magnets have attracted renewed attention as a permanent magnet material because of the supply-chain issues associated with rare earth elements [1]. The ferromagnetic $\tau$-MnAl, which has the $\mathrm{L}_{0}$ structure, has relatively good intrinsic magnetic properties $\left(\mathrm{K}_{1}=1.7 \mathrm{MJ} / \mathrm{m}^{3}\right.$, $\mathrm{M}_{\mathrm{s}}=7.5 \mathrm{kG}$ [2]), suggesting a potential maximum energy product of $\sim 12$ MGOe with appropriate microstructural development [1]. The $\mathrm{L} 1_{0}$ phase is metastable and forms from the $\varepsilon$ phase, which is stable above $870{ }^{\circ} \mathrm{C}$. The equilibrium phases at room temperature that normally result from the decomposition of the $\varepsilon$ phase are $\gamma_{2}$ and $\beta$-Mn [3].

The $\varepsilon$ phase can be stabilized at room temperature by rapid quenching from the $\varepsilon$ phase field [4-6]. This can be accomplished after conventional solidification and annealing in the $\varepsilon$ phase field, or by rapid solidification where the high cooling rate suppresses equilibrium phase formation. The compositional limit of the $\tau$ phase is essentially limited by the composition achievable in the $\varepsilon$ phase, which is reported to be $0.40<x_{\mathrm{Al}}<0.46$ [7]. The $\tau$ phase is usually formed via a moderate-temperature annealing $\left(\sim 500{ }^{\circ} \mathrm{C}\right)$, and the phase transformation proceeds via an $\varepsilon^{\prime}$ intermediate phase [8]; too high of annealing temperature results in decomposition to the $\gamma_{2}$ and $\beta$-Mn phases. The addition of $\mathrm{C}$ tends to increase the stability of the metastable $\tau$ phase, likely by suppressing formation of the equilibrium phases. The $\mathrm{C}$ addition to the $\mathrm{L} 1_{0}$ does not deleteriously affect the intrinsic properties, except for the Curie temperature, which is decreased from $392{ }^{\circ} \mathrm{C}$ to $331{ }^{\circ} \mathrm{C}$ with 1.7 atomic percent $\mathrm{C}$.

As is the case with all magnetic materials, the microstructure, particularly the grain size, plays a critical role in maximizing the extrinsic magnetic properties, namely coercivity and remanence. Phase purity additionally impacts overall saturation (and, consequently, remanence). Rapid solidification by melt spinning bypasses the primary solidification of $\gamma_{2}$ and $\beta$-Mn phases, resulting in single-phase $\varepsilon$ in the as-solidified case [5]. Further, upon conversion to the $\tau$ phase, the coercivity of melt spun $\tau$-MnAl is reported to be $1.25 \mathrm{kOe}$ [5]. Microstructural modification via mechanical milling results in a decrease in grain size, leading to increases in coercivity [9]. However, it causes formation of the $\beta$ phase which decreases the magnetization.

\section{Results and Discussion}

$\mathrm{X}$-ray diffraction of the as-melt spun material revealed single-phase $\varepsilon$, as all the diffraction peaks observed were indexed to the $\varepsilon$ phase (Figure 1). The $\varepsilon$ phase was effectively transformed to $\tau$-MnAl after heat treating at $500{ }^{\circ} \mathrm{C}$ for $10 \mathrm{~min}$, with all peaks indexing to the $\tau$ phase (Figure 1). Transmission electron microscopy showed a grain size of $\sim 500 \mathrm{~nm}$ (Figure 2), while the corresponding selected area electron diffraction pattern (inset) indexed to the $\tau$ phase, with diffraction arising from multiple grains, consistent with the X-ray diffraction results. The high-energy mechanical milling thus was initiated on either single-phase $\varepsilon$ ( $\varepsilon$-milled samples) or single-phase $\tau$ ( $\tau$-milled samples). The $\varepsilon$-milled samples were transformed to $\tau$ using the above-mentioned heat treatment. No post-milling heat treatment was done on the $\tau$-milled samples, unless specifically noted.

Mechanical milling of the initially $\tau$ phase samples resulted in a dramatic increase in the X-ray diffraction peak widths (Figure 3). Additionally, the appearance of additional diffraction maxima was observed between the two primary $\tau$ peaks $\left(\sim 45^{\circ} 2 \theta\right)$. The major $\beta$-Mn peaks are in this region, but 
the peaks are too diffuse to identify conclusively. After $3 \mathrm{~h}$ of milling, the $\tau$ peaks are very broad, and further high-energy mechanical milling results in amorphization. For the $\varepsilon$-milled samples, the peak widths of the $\tau$ phase (note that the sequence was milling in the $\varepsilon$ phase, followed by conversion to $\tau$ at $500{ }^{\circ} \mathrm{C}$ for $10 \mathrm{~min}$ ) were notably more narrow (Figure 4). The increased peak widths of the $\tau$-milled samples were due to both grain size reduction and increased structural disorder induced by the mechanical milling (notably, strain). Conversely, the $\tau$ phase formed in the $\varepsilon$-milled samples would lack the structural disorder induced by milling, similar to nearly defect-free grains that result from recrystallization, and the breadth of the diffraction peaks would solely be due to grain size effects. A secondary phase also evolves in the $\varepsilon$-milled samples, and in this case the peaks were positively identified to be from $\beta-\mathrm{Mn}$.

Figure 1. X-ray diffraction patterns for (a) as-spun $\mathrm{Mn}_{54} \mathrm{Al}_{43} \mathrm{C}_{3}$, and (b) after heat treatment at $500{ }^{\circ} \mathrm{C}$ for $10 \mathrm{~min}$.

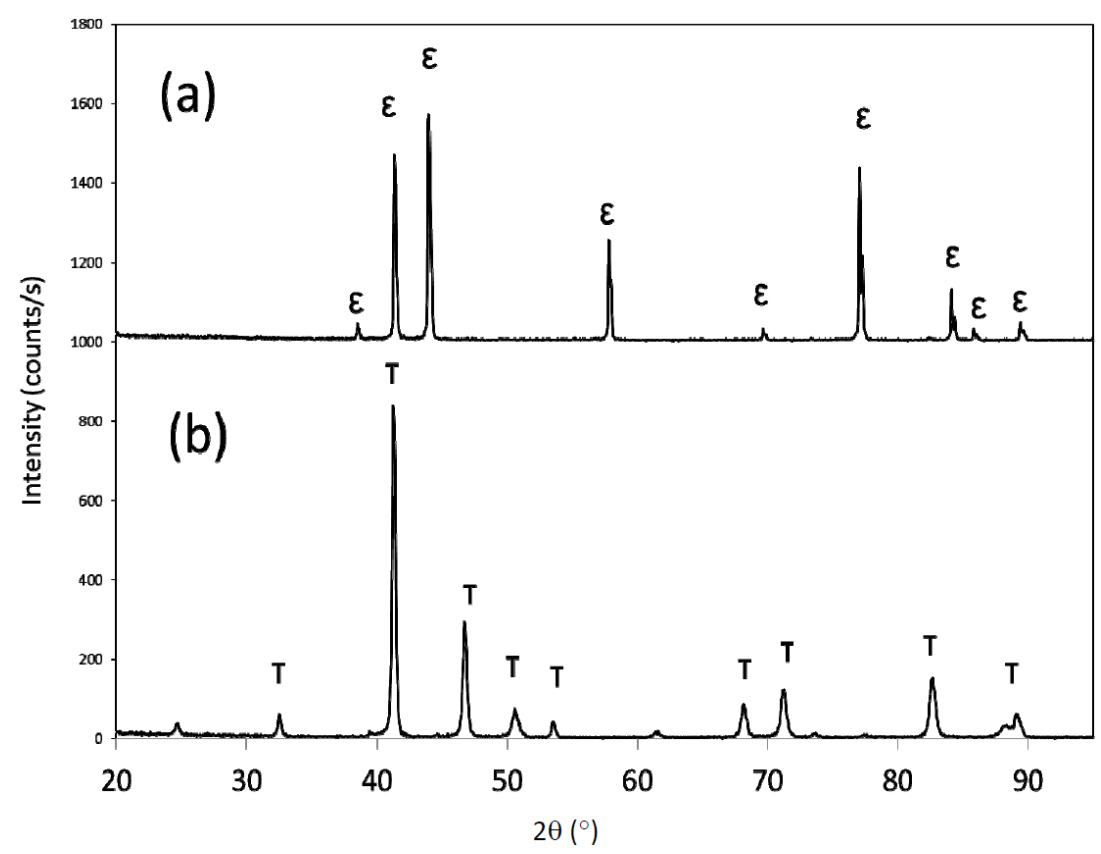

Figure 2. Transmission electron micrograph of melt spun Al-Mn-C after conversion to $\tau$ (inset: Selected area electron diffraction pattern of the region).

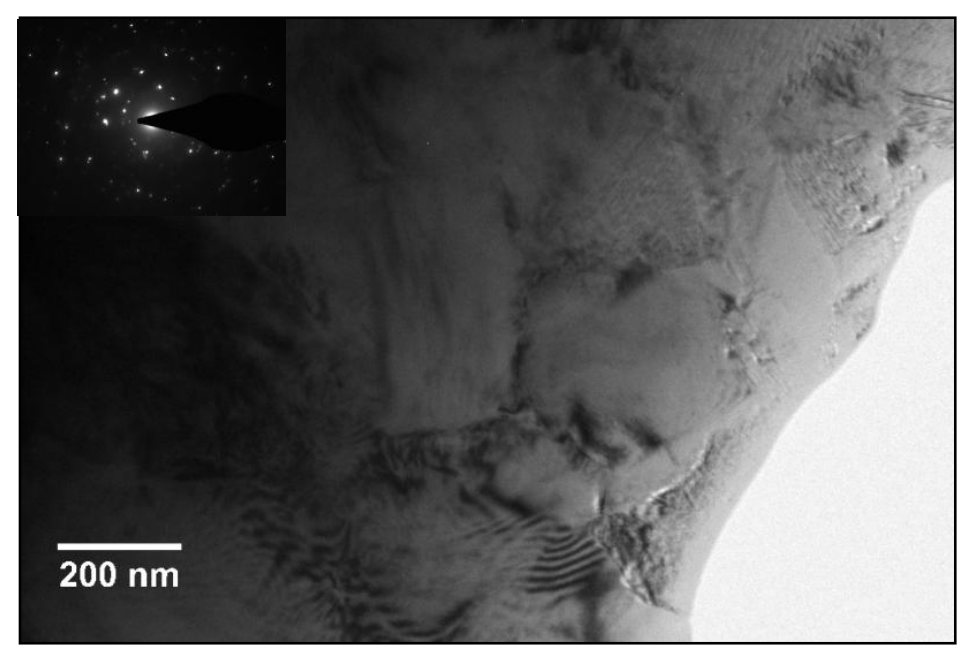


Figure 3. X-ray diffraction patterns for $\tau$-milled samples for different milling (MM) times. The bottom pattern is as-annealed (initial $\tau$ phase).

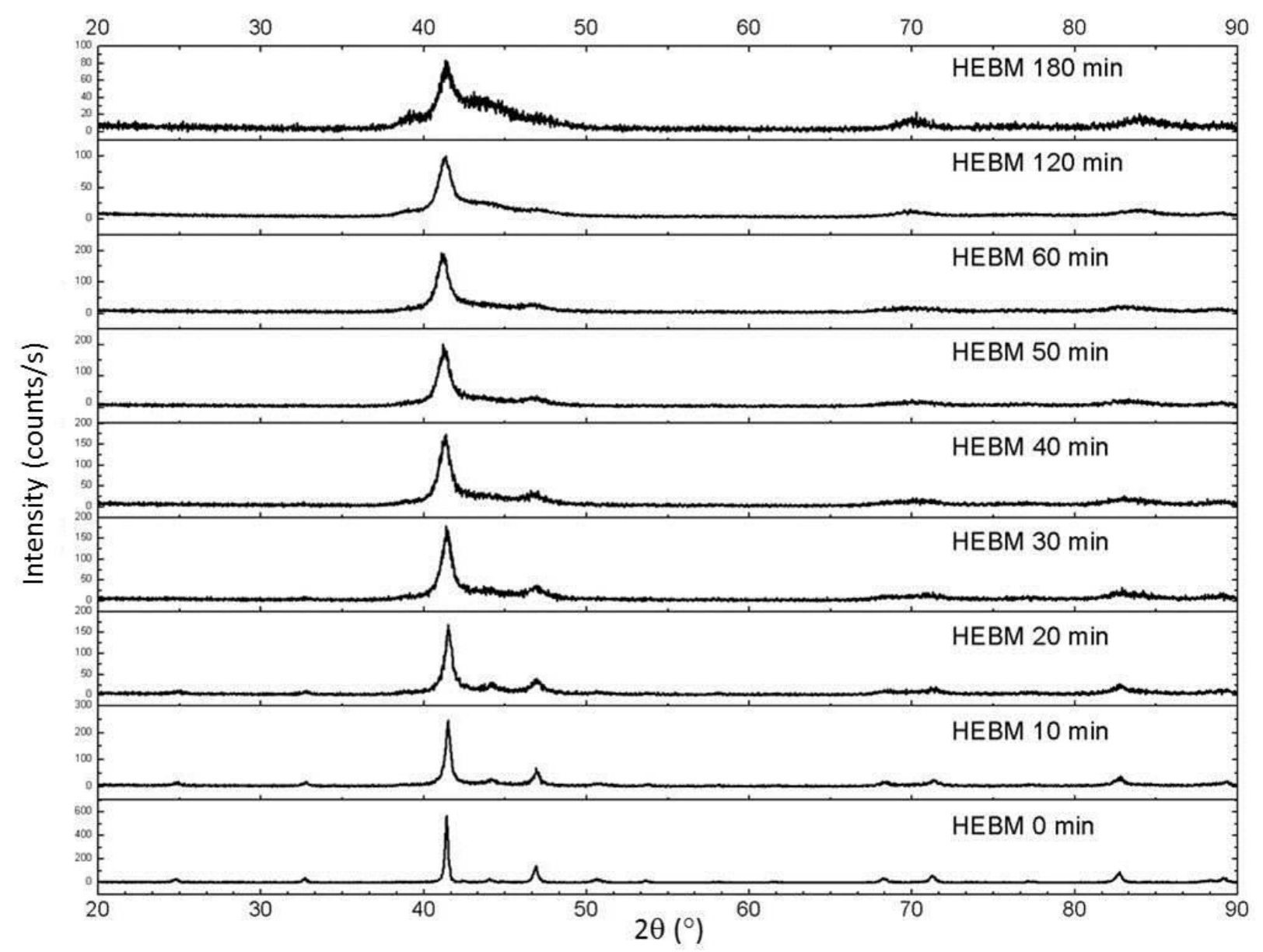

Figure 4. X-ray diffraction patterns for $\varepsilon$-milled samples after conversion from $\varepsilon$ to $\tau$.

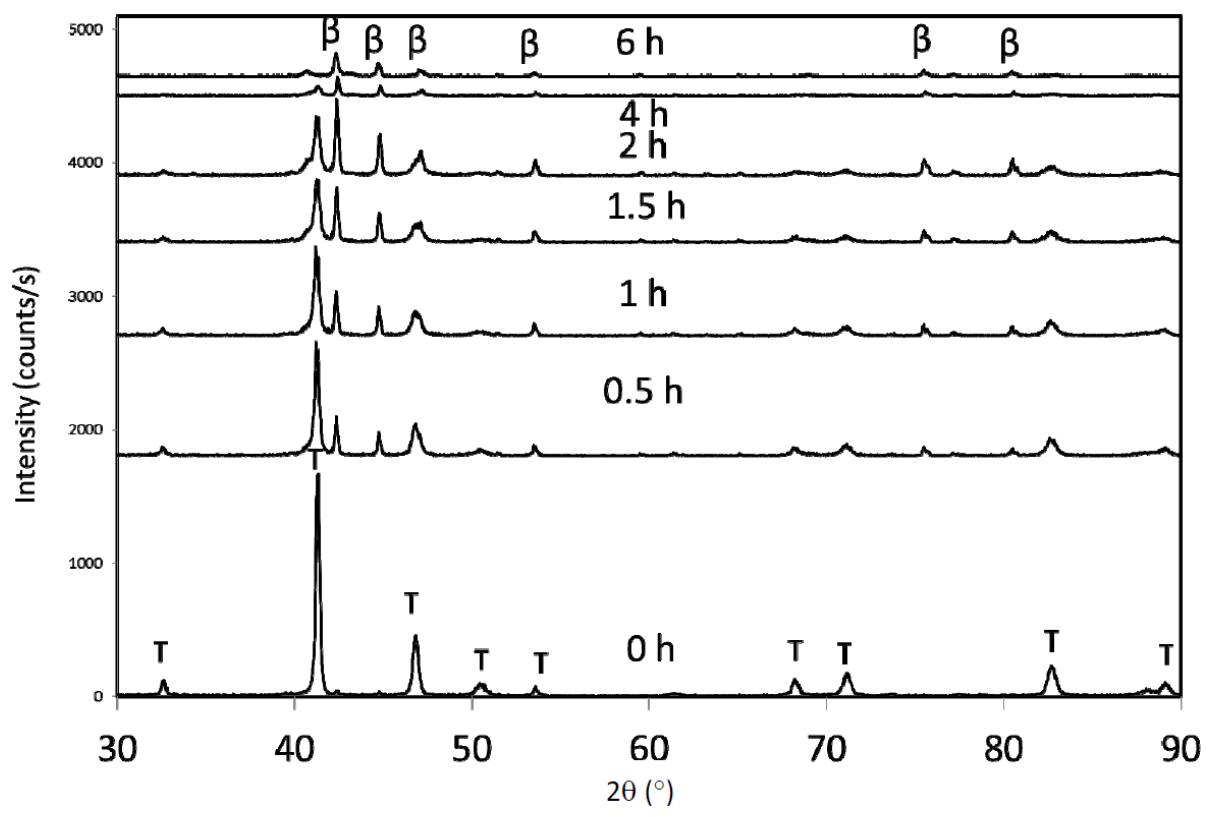

In both $\varepsilon$-milling and $\tau$-milling, the high-energy mechanical milling resulted in a significant increase in the coercivity (Figure 5). Also in both cases, the initial increase in coercivity was followed 
by a subsequent decrease upon further milling (Figure 6). The maximum coercivity was similar in both cases (4.6 kOe for $\tau$-phase milling, $4.1 \mathrm{kOe}$ for $\varepsilon$-phase milling). Additionally, the maximum was at similar milling times ( $1 \mathrm{~h}$ and $1.5 \mathrm{~h}$, respectively). However, the coercivity of the $\varepsilon$-milled samples did not decrease as dramatically with milling time. The coercivity decrease in the $\tau$-milled samples is likely due to amorphization, where the amorphous phase is magnetically soft. In both cases, a dramatic loss of magnetization was also observed. The loss in magnetization is much more significant in the $\tau$-milled samples, but is also dramatic in the $\varepsilon$-milled samples.

Figure 5. (a) Hysteresis loops for $\varepsilon$ phase-milled samples for different milling times, after conversion of $\varepsilon$ to $\tau$; (b) Hysteresis loops for samples milled in the $\tau$ phase.
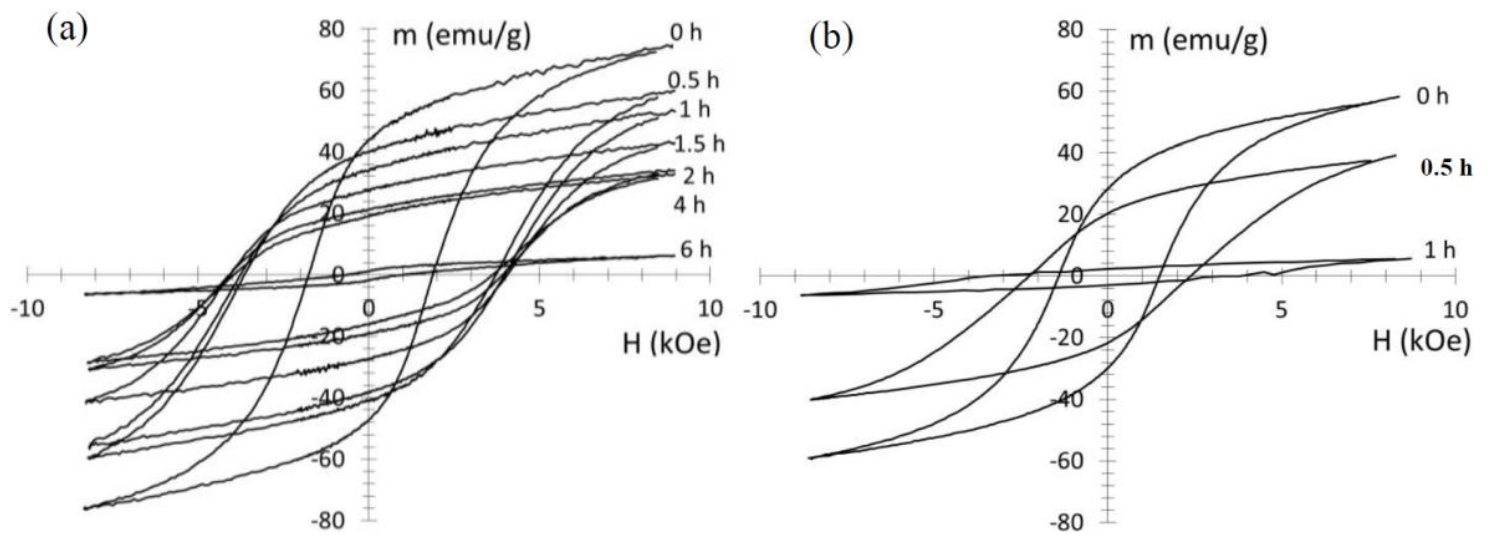

Figure 6. Relationship between coercivity and milling time and magnetization (at $8 \mathrm{kOe}$ ) and milling time for both the (a) $\varepsilon$ milled (solid line), and (b) $\tau$ milled samples (dashed line).
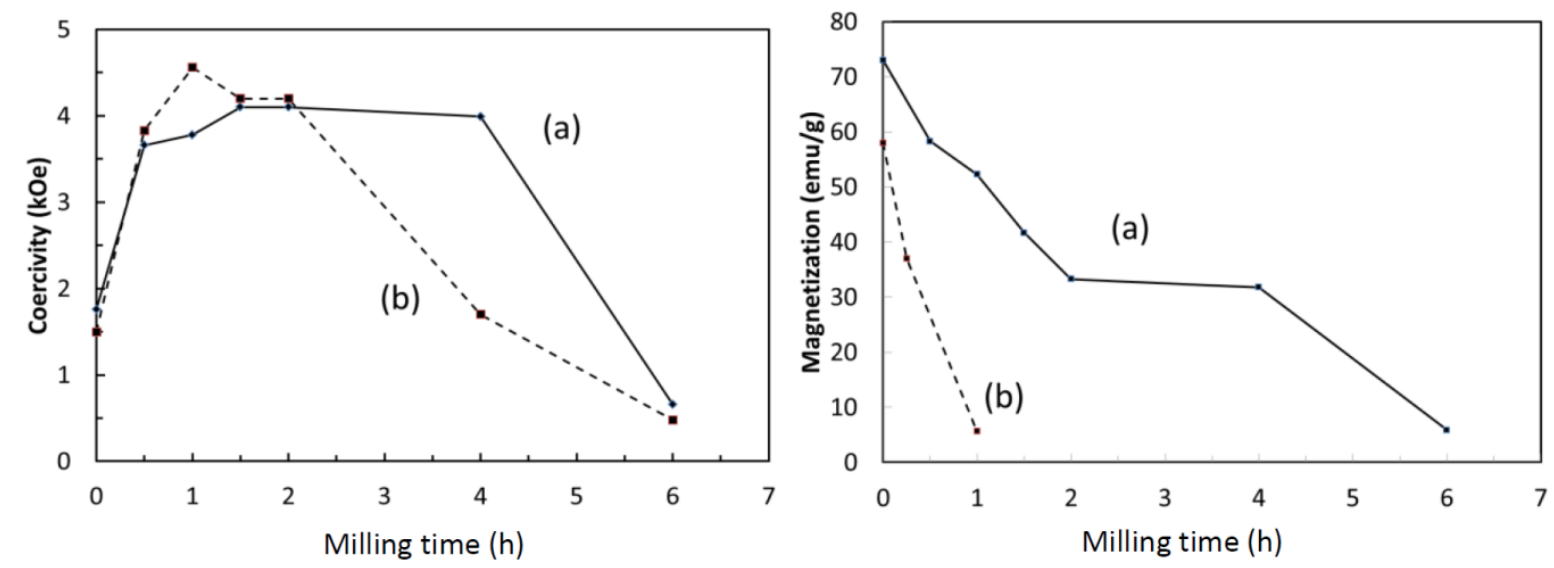

The loss of magnetization with milling time may be attributed to the formation of the $\beta$-Mn phase, which reduced the phase fraction of the ferromagnetic $\tau$ phase. However, the loss in magnetization appeared more significant than the phase fraction of the $\beta$-Mn, particularly for the $\tau$-milled sample. The loss of magnetization could possibly result from oxidation during milling, or from site disorder induced by the milling of the $\tau$ phase. The latter is essentially a loss of the long-range order, and the resulting antisite disorder resulting in antiferromagnetic coupling between $\mathrm{Mn}$ atoms along the c-direction.

If oxidation occurred during milling, then an effective change in $\mathrm{Mn}$ :Al ratio would result and the phase relationships would be altered. Thus, if a milled sample is annealed in the $\varepsilon$ phase field (above $1050{ }^{\circ} \mathrm{C}$ ), then the phase transformation to the $\beta$ phase can be reversed back to the $\varepsilon$ phase, and 
ultimately form the $\tau$ phase. If oxidation occurred, then the composition would shift so that it would no longer be in the $\varepsilon$ single-phase field. Figure 7 shows a sample that was milled, annealed at $1050{ }^{\circ} \mathrm{C}$ for $10 \mathrm{~min}$, and then annealed at $500{ }^{\circ} \mathrm{C}$ for $10 \mathrm{~min}$. The XRD pattern revealed single-phase $\tau$, suggesting that no significant change in composition occurred that resulted in the formation of $\beta$-Mn during milling (Figure 5). Interestingly, the original coercivity was recovered upon conversion to $\tau$. Presumably, the grain size increased as a result of the high temperature heat treatment but with no effect on the coercivity. Additionally, energy dispersive X-ray spectroscopy revealed only trace oxygen levels up to $1 \mathrm{~h}$ of milling, although long-term milled samples (10 h) showed significant oxidation [6]. It should be noted that the mechanical milling protocol of the latter sample was different, notably that intermittent milling was not employed.

Figure 7. X-ray diffraction pattern (a) $1 \mathrm{~h} \varepsilon$-milled sample, converted to $\tau$ showing significant phase fraction of $\beta-\mathrm{Mn}$, and (b) same sample after annealing at $1050{ }^{\circ} \mathrm{C}$ to transform the material back to $\varepsilon$, and then conversion to $\tau$ at $500{ }^{\circ} \mathrm{C}$ for $10 \mathrm{~min}$. It returns to single-phase $\tau$.

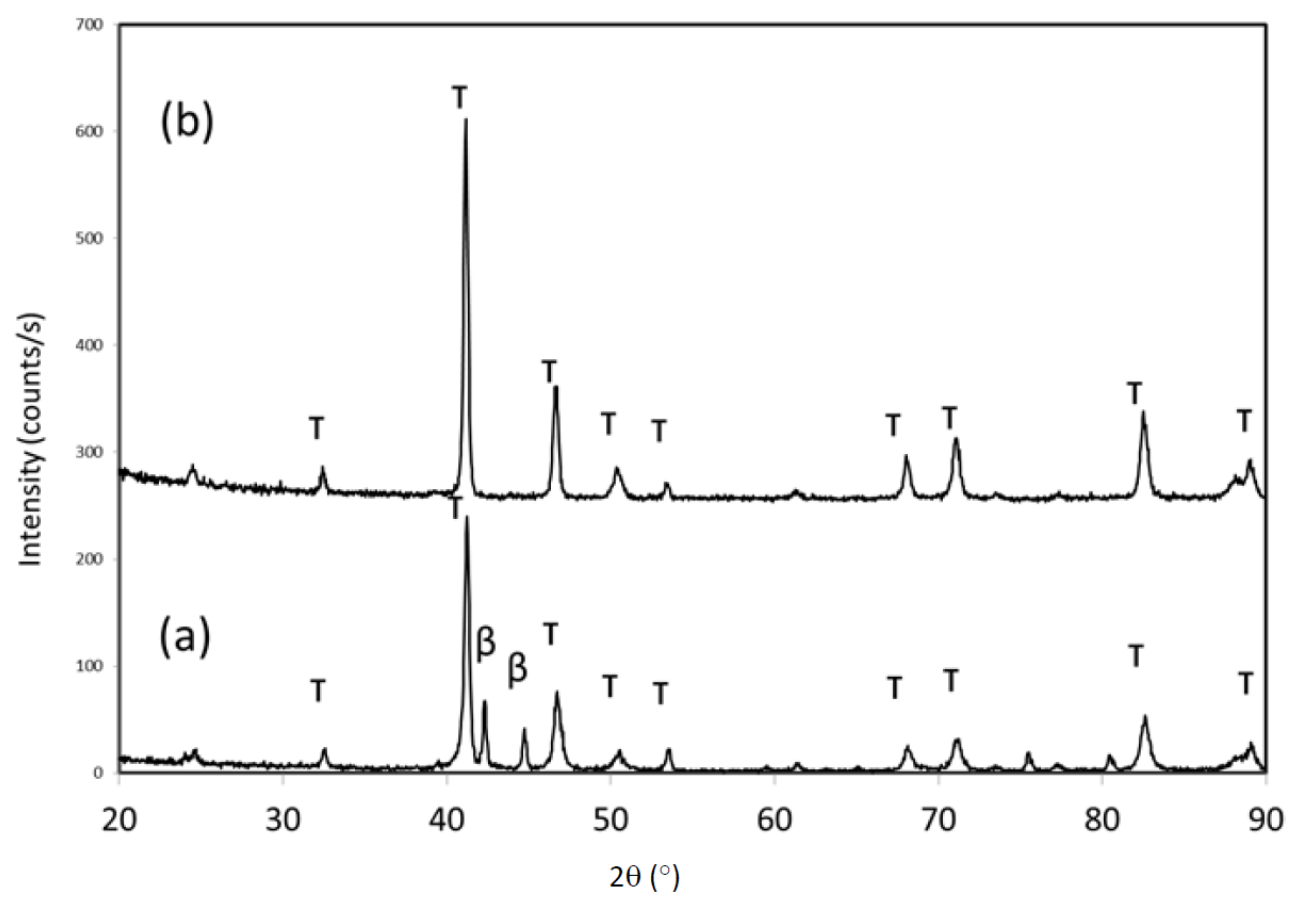

In order to determine if site disorder was responsible for the loss of magnetization, a sample was milled for $15 \mathrm{~min}$ and the intensity ratio $\mathrm{I}_{(001)} / \mathrm{I}_{(111)}$ was compared to the original intensity. If site disorder increased, then the intensity of the (001) superlattice peak would decrease (relative to the (111) fundamental peak). The peaks were fitted with a Gaussian fit to determine the area. Figure 8 shows the XRD data and the Gaussian fits. The intensity ratio of the 15 min milled sample was virtually identical to the non-milled sample (both $0.6 \pm 0.05$ ), indicating that site disorder did not increase upon milling. It should be noted that a $\sim 15 \%$ loss in magnetization was observed even with the relatively short-term milling.

With neither oxidation nor site disorder contributing to the loss of magnetization, it appears that the formation of the equilibrium $\beta$-Mn (and presumably $\gamma_{2}$ below detection limits) is the predominant contributor. As mentioned, the nominal composition of the alloy lies in the equilibrium phase field of 
$\beta$-Mn $+\gamma_{2}$; thus any decomposition of the metastable $\tau$ phase, or equilibrium decomposition of the $\varepsilon$ phase, would result in the formation of these phases. Since neither of these phases is ferromagnetic (or ferrimagnetic), then their presence would reduce the phase fraction of the ferromagnetic $\tau$ phase, and lower magnetization. Since the high-energy mechanical milling was done using short term (1 min) intermittent "pulses" to alleviate temperature excursions, the transformation to the equilibrium phases is likely stress-induced. This is similar to what has been observed in other systems, where crystallization of amorphous structures (i.e., transformation from metastable to stable structures) was stress-induced [10]. The crystallization in this case is thought to be enabled by an increase in defect density [11]. However, it is also probable that some local heating occurred, even with the short-term milling, which assisted in the phase transformation.

Figure 8. X-ray diffraction data and Gaussian fits of the (001) and (111) peaks for (a) unmilled and (b) 15 min milled samples. The numbers shown are areas (in arbitrary units).

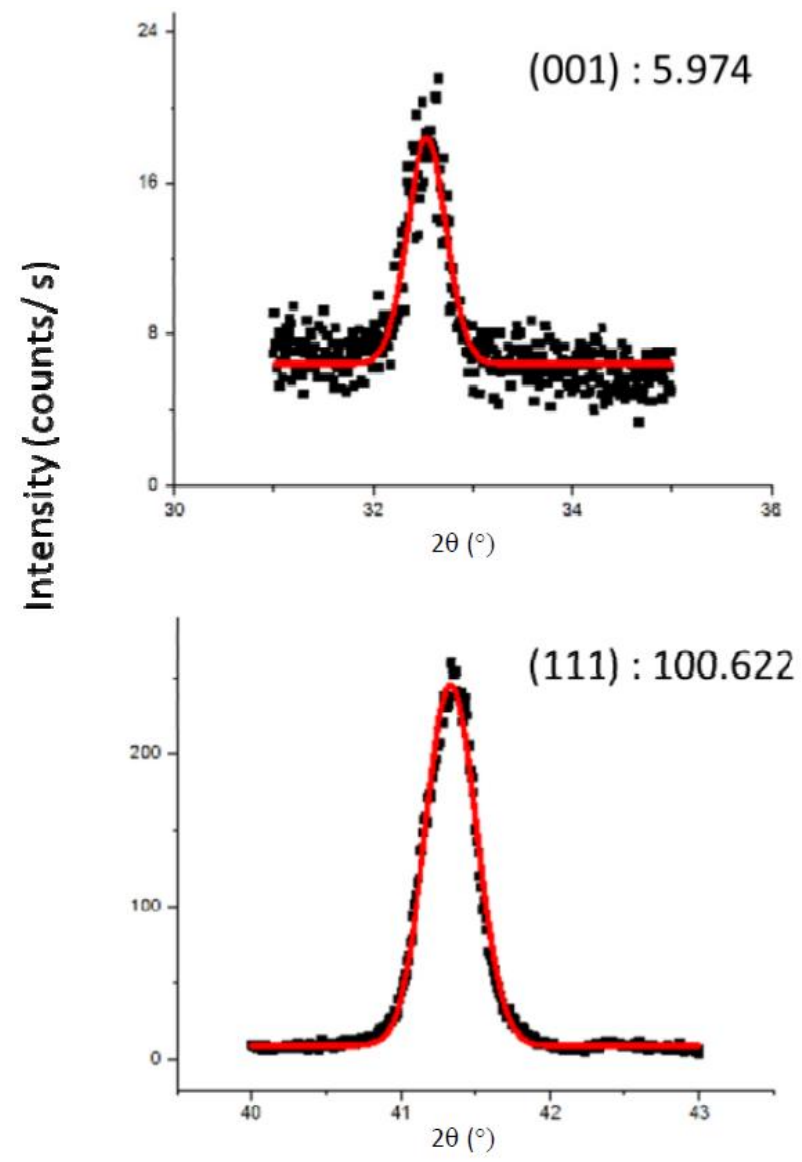

(a)
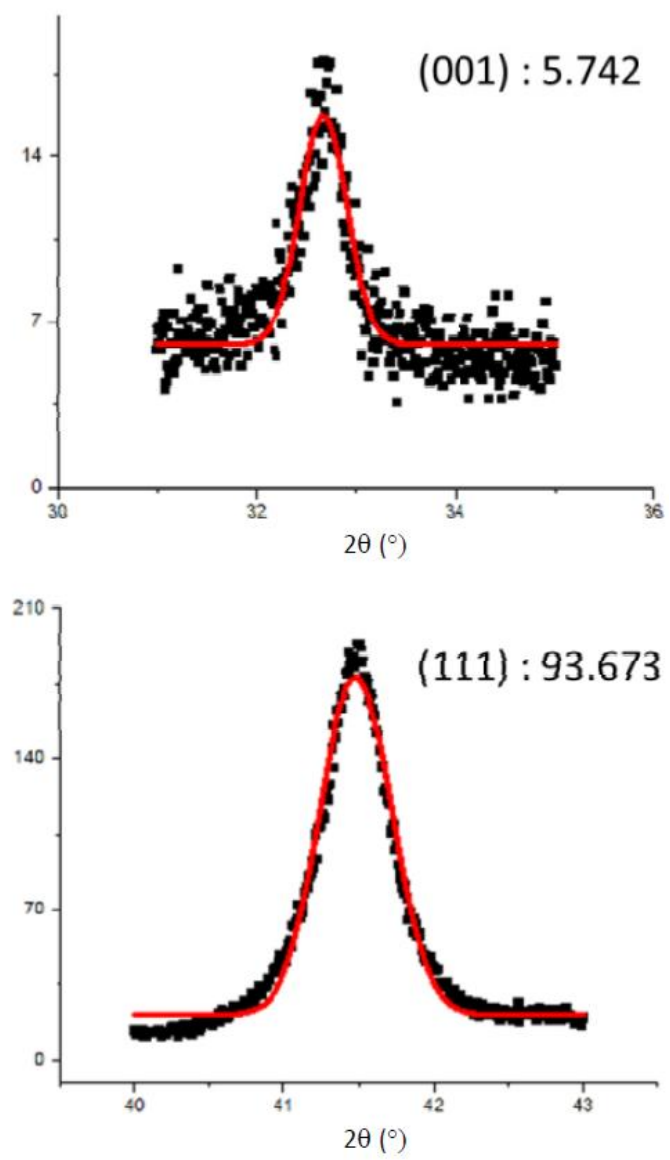

(b)

$\tau$-phase samples were also mechanically milled using a surfactant (surfactant-assisted mechanically milling). The surfactant eliminates re-welding of fractured particles that occurs during dry milling. This consequently leads to a reduction in particle size [12-16]. Figure 9 shows X-ray diffraction patterns of the surfactant-assisted milled samples. Note that significant broadening occurs, but even at long milling times amorphization does not occur (compare this behavior with dry milling, shown in Figure 3). Also, the predominant phase remains $\tau$, with a very small fraction of equilibrium phases. The coercivity behavior is similar to the dry-milled samples, with an increase with milling time 
(Figure 10). Here, however, the dramatic loss of coercivity observed in the dry-milled samples does not occur, as a relatively stable coercivity results even for times up to $32 \mathrm{~h}$. The decrease in magnetization also occurs here, however, but the decrease is less dramatic than in the dry-milled $\tau$ phase samples, and more along the lines of the $\varepsilon$-milled samples. The morphology of the milled samples was interesting as well. After $16 \mathrm{~h}$ of milling, the particles consisted of very flat platelets with a tremendously high aspect ratio. The thickness of the plates after milling for $16 \mathrm{~h}$ is on the order of tens of nanometers, while the length and width are on the order of several microns (Figure 11). Presumably, the X-ray peak broadening arises due to preferential alignment of the particles during sample preparation for X-ray diffraction (i.e., they lie flat on the sample holder so size broadening arises from the particle thickness). Interestingly, the fracture of the particles appears to be mica-like (that is, in sheets). Typically, fracture occurs either transgranular or intergranular. The microstructure (Figure 2) consists of predominantly equiaxed grains, so the exact fracture behavior of the melt spun ribbon is not clear. This is in contrast to surfactant-assisted milling studies in other systems, which produced distinct nanoparticles after similar times [12-16].

Figure 9. X-ray diffraction patterns of surfactant-assisted mechanical milling (SAMM) as a function of milling time.

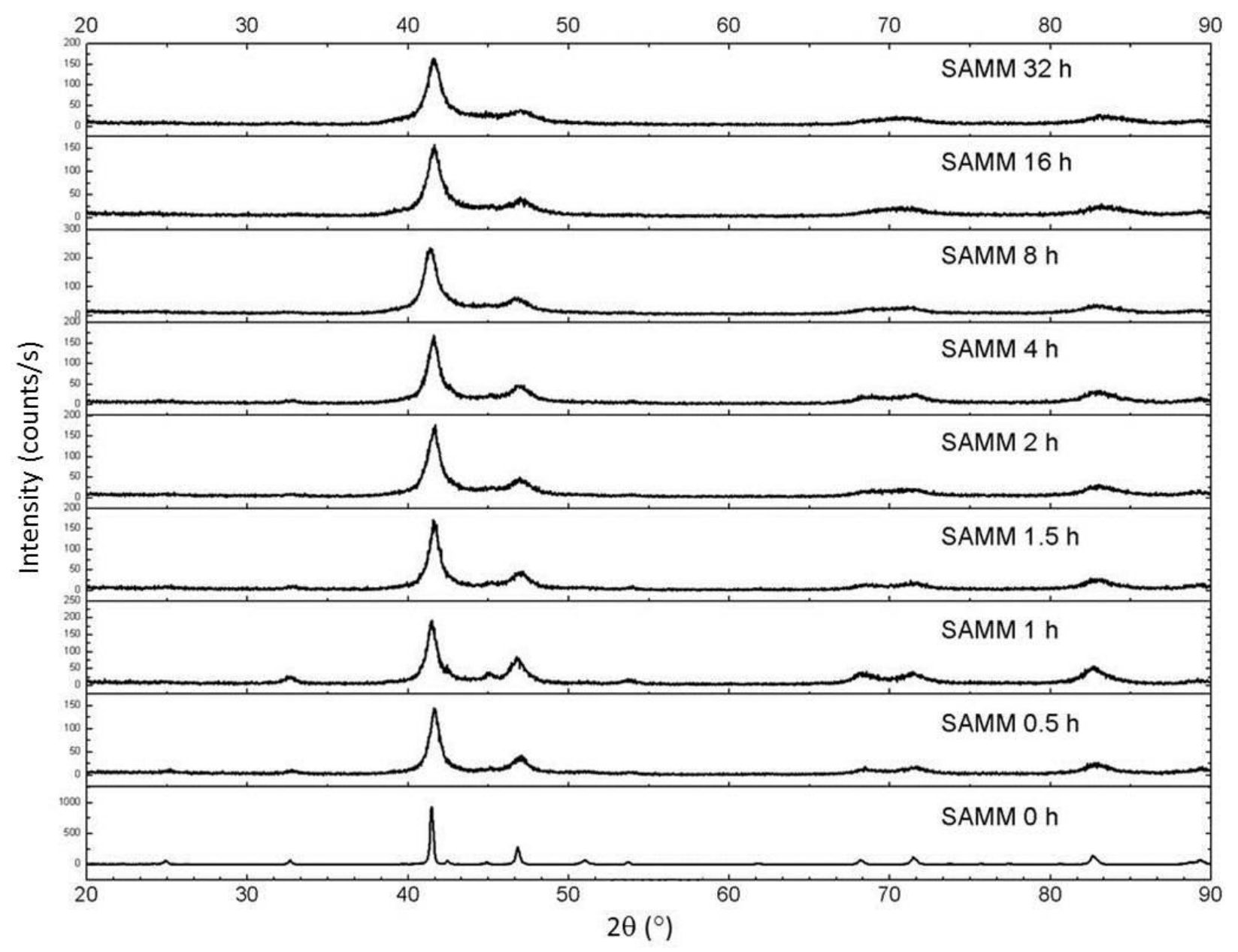


Figure 10. Relationship between surfactant-assisted milling time and coercivity.

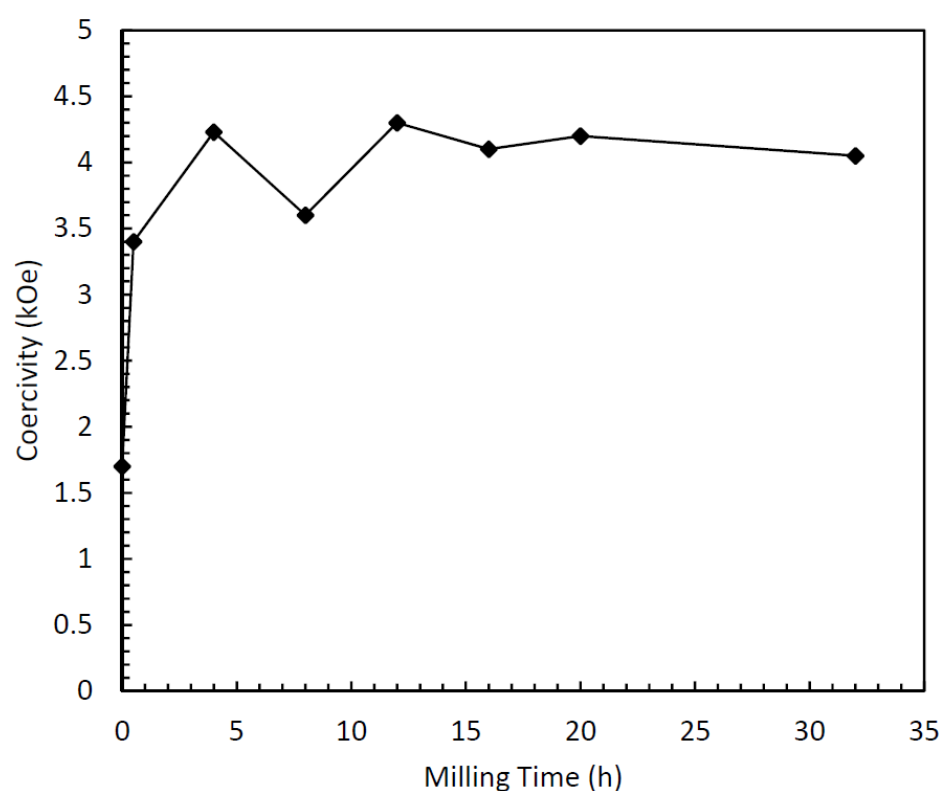

Figure 11. Scanning electron micrograph showing the particle morphology of surfactant-assisted mechanically milled Mn-Al-C particles.

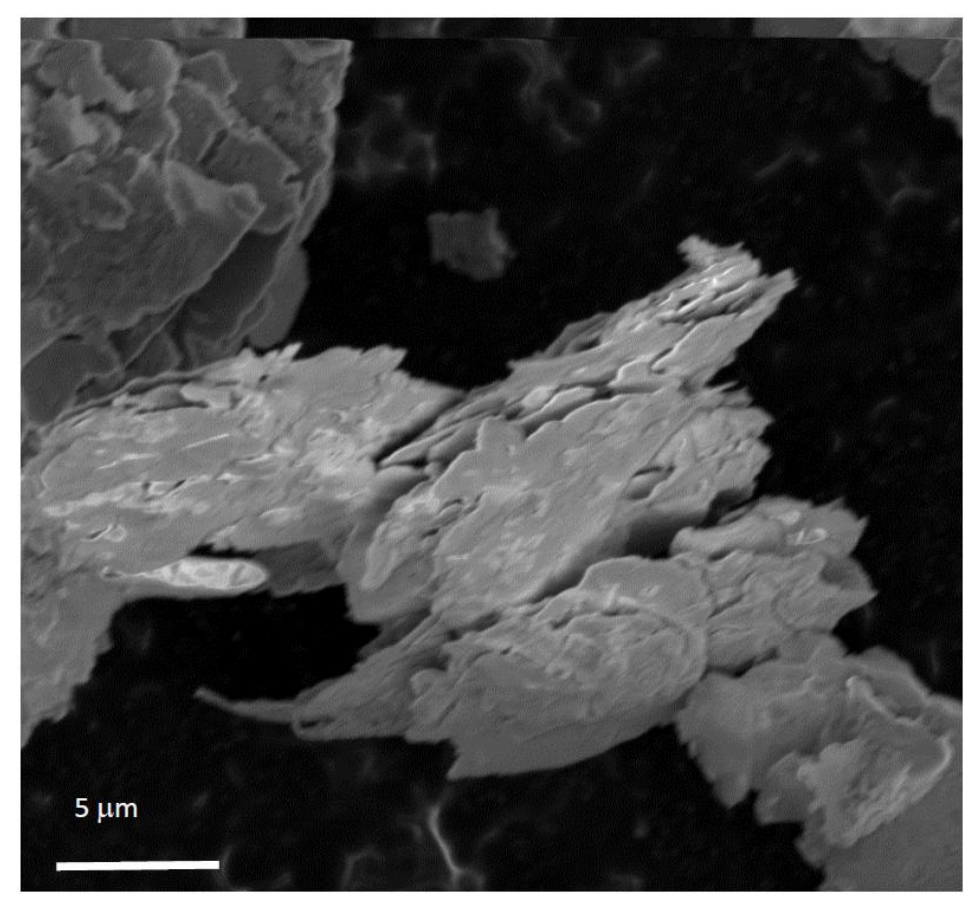

\section{Experimental Section}

The composition of $\mathrm{Mn}_{54} \mathrm{Al}_{43} \mathrm{C}_{3}$ was chosen to maximize $\tau$ phase fraction. Alloys were obtained by arc melting of high purity elements ( $>99.9 \%$ purity). The ingot was then melt spun at a tangential speed of $40 \mathrm{~m} / \mathrm{s}$. The materials were heat treated at $500{ }^{\circ} \mathrm{C}$ for $10 \mathrm{~min}$ after sealing under UHP Ar. Samples were quenched in water from the heat treatment temperature. Mechanical milling was done in a SPEX 8000 Mixer/Mill (SPEX Sample Prep, Metuchen, NJ, USA) using hardened steel vials and balls. The Mn-Al-C alloys were loaded into the vials and sealed in a nitrogen atmosphere glove box. 
Milling was done on an intermittent basis of one minute on followed by one minute off to reduce temperature excursions. Surfactant-assisted mechanical milling was accomplished using oleic acid (powder-to-oleic acid ratio of 20:1) and heptane as the medium. In this case, the milling protocol was $10 \mathrm{~min}$ on, 5 min off. X-ray diffraction (XRD) analysis was done using a Rigaku Multiflex (Rigaku Corporation, Tokyo, Japan) with $\mathrm{Cu} \mathrm{K \alpha}$ radiation and a post-sample graphite monochromator. Powdered samples were placed on an off-cut $\mathrm{SiO}_{2}$ single crystal to minimize diffraction affects from the holder. Magnetic measurements were completed using a Lakeshore 8500 VSM (Lakeshore Cryotonics, Westerville, OH, USA) and were conducted at room temperature with a maximum field of 8 kOe.

\section{Conclusions}

Two-step processing involving melt spinning followed by high-energy mechanical milling results in increased coercivity but a loss of magnetization for $\mathrm{Mn}_{54} \mathrm{Al}_{43} \mathrm{C}_{3}$ alloys. The milling was accomplished on material starting in both the $\varepsilon$ phase, which is formed upon melt spinning, and the $\tau$ phase, formed upon heat treatment of $\varepsilon$ at $500{ }^{\circ} \mathrm{C}$ for $10 \mathrm{~min}$. While significant coercivity values were obtained (>4 kOe in both cases), a loss of magnetization with milling time was observed. The loss of magnetization was more significant for $\tau$ phase milling. In both cases the magnetization loss was attributed to stress-induced formation of room-temperature stable phases, notably $\beta$-Mn. No increase in site disorder was induced by short-term milling, and phase formation induced by milling was reversible. Surfactant-assisted milling resulted in platelet-like particles and similar increases in coercivity as observed in the dry milling.

\section{Acknowledgments}

The authors gratefully acknowledge research support from the Department of Energy through the ARPA-E REACT program under Grant No. 0472-1537. The authors are also grateful for facility support from the Nebraska Research Initiative through the Nebraska Center for Materials and Nanoscience at the University of Nebraska.

\section{Author Contributions}

Michael Lucis, Timothy Prost and MeiyuWang conducted the experiments, collected data, and interpreted results. Xiujuan Jiang contributed the electron microscopy, including sample preparation, image and diffraction collection, and interpretation/analysis. Jeffrey Shield directed the research, interpreted results, and prepared the manuscript.

\section{Conflicts of Interest}

The authors declare no conflict of interest.

\section{References}

1. Skomski, R.; Manchanda, P.; Kumar, P.; Balamurugan, B.; Kashyap, A.; Sellmyer, D.J. Predicting the future of permanent-magnet materials. IEEE Trans. Magn. 2013, 49, 3215-3220. 
2. Skomski, R.; Coey, J.M.D. Permanent Magnetism; Institute of Physics Publishing: Bristol, UK, 1999.

3. Massalski, T.B. Binary Alloy Phase Diagrams, 2nd ed.; ASM International: Materials Park, $\mathrm{OH}$, USA, 1990; Volume 1.

4. Liu, Z.W.; Chen, C.; Zheng, Z.G.; Tan, B.H.; Ramanujan, R.V. Phase transitions and hard magnetic properties for rapidly solidified $\mathrm{MnAl}$ alloys doped with $\mathrm{C}, \mathrm{B}$, and rare earth elements. J. Mater. Sci. 2012, 47, 2333-2338.

5. Fazakas, E.; Varga, L.K.; Mazaleyrat, F. Preparation of nanocrystalline Mn-Al-C magnets by melt spinning and subsequent heat treatments. J. Alloys Compd. 2007, 434-435, 611-613.

6. Le Breton, J.M.; Bran, J.; Folcke, E.; Lucis, M.; Lardé, R.; Jean, M.; Shield, J.E. Structural modifications in a $\mathrm{Mn}_{54} \mathrm{Al}_{43} \mathrm{C}_{3}$ melt-spun alloy induced by mechanical milling and subsequent annealing investigated by atom probe tomography. J. Alloys Compd. 2013, 581, 86-90.

7. Ellner, M. Crystal chemical parameters of the liquid-quenched $\mathrm{MnAl}_{0.8}$. J. Alloys Compd. 2006, 422, 192-193.

8. Yanar, C.; Wiezorek, J.; Radmilovic, V.; Soffa, W.A. Massive transformation and the formation of the ferromagnetic $\mathrm{L}_{0}$ phase in manganese-aluminum-based alloys. Metall. Mater. Trans. A 2002, 33, 2413-2423.

9. Zeng, Q.; Baker, I.; Cui, J.B.; Yan, Z.C. Structural and magnetic properties of nanostructured Mn-Al-C magnetic materials. J. Magn. Magn. Mater. 2007, 308, 214-226.

10. Ma, E.; Atzmon, M. Phase transformations induced by mechanical alloying in binary systems. Mater. Chem. Phys. 1995, 39, 249-267.

11. Geng, Y. High-Energy Mechanical Milling-induced Crystallization in $\mathrm{Fe}_{32} \mathrm{Ni}_{52} \mathrm{Zr}_{3} \mathrm{~B}_{13}$. unpublished work.

12. Poudyal, N.; Rong, C.; Liu, J.P. Effects of particle size and composition on coercivity of Sm-Co nanoparticles prepared by surfactant-assisted ball milling. J. Appl. Phys. 2010, 107, 09A703.

13. Poudyal, N.; Nguyen, V.; Rong, C.; Liu, J.P. Anisotropic bonded magnets fabricated via surfactant-assisted ball milling and magnetic-field processing. J. Phys. D 2011, 44, 335002.

14. Akdogan, N.; Hadjipanayis, G.; Sellmyer, D. Novel $\mathrm{Nd}_{2} \mathrm{Fe}_{14} \mathrm{~B}$ nanoflakes and nanoparticles for the development of high energy nanocomposite magnets. Nanotechnology 2010, 21, 295705.

15. Su, K.P.; Liu, Z.W.; Zeng, D.C.; Huo, D.X.; Li, L.W.; Zhang, G.Q. Structure and size-dependent properties of $\mathrm{NdFeB}$ nanoparticles and textured nano-flakes prepared from nanocrystalline ribbons. J. Phys. D 2013, 46, 245003.

16. Cui, B.Z.; Zheng, L.Y.; Li, W.F.; Liu, J.F.; Hadjipanayis, G.C. Single-crystal and textured polycrystalline $\mathrm{Nd}_{2} \mathrm{Fe}_{14} \mathrm{~B}$ flakes with a submicron or nanosize thickness. Acta Mater. 2012, 60, 1721-1730.

(C) 2014 by the authors; licensee MDPI, Basel, Switzerland. This article is an open access article distributed under the terms and conditions of the Creative Commons Attribution license (http://creativecommons.org/licenses/by/3.0/). 